\title{
EXCLUSIÓN, DISCRIMINACIÓN Y DISCAPACIDAD: EL DISCURSO SOBRE LA DISCAPACIDAD EN ACTAS DE LA ASAMBLEA LEGISLATIVA DE COSTA RICA
}

\section{EXCLUSION, DISCRIMINATION AND DISABILITY: DISCOURSE ABOUT DISABILITY IN THE PLENARY SESSION OF THE COSTA RICAN LEGISLATIVE ASSEMBLY}

\section{Adrián Vergara Heidke*}

\section{RESUMEN}

En el presente artículo se busca analizar el discurso sobre la discapacidad que se manifiesta en Actas de Sesiones del Plenario de la Asamblea Legislativa de Costa Rica, con el fin de identificar aquellos componentes que puedan resultar excluyentes y discriminatorios para las personas con discapacidad. Se aplican fundamentos teóricos y categorías metodológicas del análisis crítico del discurso. Los resultados evidencian construcciones discriminatorias y menospreciativas hacia las personas con discapacidad por parte de diputados y diputadas.

PALABRAS CLAVE: DISCURSO * ANÁLISIS LINGÜISTICO * DISCAPACIDAD * POLÍTICA * PODER LEGISLATIVO

ABSTRACT

The objective of this article is to analyze the discourse of disability that is expressed in the Plenary Session of the Costa Rican Legislative Assembly, in order to identify those components that may be exclusionary and discriminatory for people with disabilities. Theoretical foundations and methodological categories of critical discourse analysis have been applied. The results show discriminatory and disparaging constructions towards people with disabilities by Legislative Assembly members.

KEYWORDS: SPEECHES $*$ LINGUISTIC ANALYSIS * DISABILITY * POLITIC * LEGISLATIVE POWER 


\section{INTRODUCCIÓN}

En el año 2007, varios países firmaron la Convención Internacional sobre los Derechos de las Personas con Discapacidad en la ONU. Entre esos países, se encontraba Costa Rica, cuya Asamblea Legislativa aprobó la Convención y su Protocolo Facultativo el 7 de agosto de 2008. Este texto se basa en el modelo social de la discapacidad que la considera como una construcción a partir de la relación entre el entorno situacional y social, así como, personas con deficiencias físicas, mentales, intelectuales o sensoriales.

En este contexto, se ha propuesto estudiar los discursos sobre la discapacidad que existen en Costa Rica ${ }^{1}$, con el fin de identificar los componentes o los discursos excluyentes y discriminatorios hacia las personas con discapacidad (o con diversidad funcional). La perspectiva teórica es el análisis crítico del discurso (ACD). Se considera que el ACD es una perspectiva aplicada dentro del análisis del discurso que aborda críticamente los discursos y sus relaciones con realidades sociales. Esta crítica se lleva a cabo desde un posicionamiento políticomoral, en el que se tiene una concepción de las personas como seres sociales y se busca una sociedad donde convivan solidariamente las personas, distintos grupos sociales y discursos alternativos. Este posicionamiento provoca inconformidad respecto a discursos y realidades sociales que atenten contra estos postulados, los cuales se intentan transformar.

En este texto se tiene por objetivos, por un lado, exponer la base teórica y conceptualización de discurso y por otro lado, evidenciar los componentes de un discurso sobre la discapacidad excluyente $y$ discriminatorio en fragmentos de dos Actas del Plenario de la Asamblea Legislativa de Costa Rica durante la discusión y aprobación de la Convención Internacional sobre los Derechos de las Personas

1 Este texto es producto de un proyecto de investigación más amplio "El discurso sobre la discapacidad en Costa Rica: el discurso sobre la discapacidad en ámbitos cotidianos, educativos y políticos" inscrito en el Instituto de Investigaciones Lingüísticas de la Universidad de Costa Rica. con Discapacidad. Uno de los aspectos relevantes de analizar estos textos es que pertenecen a un género textual planificado y normado socialmente, en especial, por lo "políticamente correcto", lo que hace difícil que aparezcan esos componentes excluyentes $y$ discriminatorios.

En una primera parte de este trabajo, se exponen los presupuestos teóricos y, luego, a partir de ellos, la conceptualización de discurso y su relación con las interacciones sociales, entre las cuales se encuentran las comunicativas. A continuación, se explica sucintamente el modelo social de la discapacidad. Finalmente, se desarrolla un análisis de esos componentes de un discurso sobre la discapacidad excluyente $y$ discriminatorio que se manifiesta en los textos seleccionados.

\section{REFERENCIA TEÓRICA}

\section{CONCEPTO DE DISCURSO}

Como se presenta en Vergara (2016), a partir de unos presupuestos teóricos explícitos, se define el discurso como "el conocimiento sobre alguna cosa, el cual se considera como verdadero por un grupo de personas que lo "comparten", además, guía nuestros actos y nunca se encuentra aislado, ya que tiene relación con otros conocimientos sobre otras cosas" (p.244).

A continuación, se explicará esta definición en detalle. El discurso es el conocimiento sobre alguna cosa, expresión redundante si se considera que siempre el conocimiento será sobre algo, sin embargo, ese algo no se refiere al "original" de las representaciones (de qué cosa es una representación), sino a un contenido específico que es delimitado por el personal científico que aborde un discurso (Busse y Wolfgang, 1994). Sobre qué versa el discurso o sobre qué discurso se trabajará o hablará constituye una decisión científica. Cada investigador o investigadora definirá a partir de criterios como intereses, puntos de vista, objetivos, ese algo. De esta manera, esas representaciones y asociaciones (red de representaciones), que es el conocimiento, aparecen agrupadas según 
criterios científicos ${ }^{2}$ que deben ser justificados. Es ese algo lo que permite que esas representaciones estén asociadas entre sí en un momento dado. Esto no significa que ese conjunto de representaciones $y$ asociaciones sea un producto científico (constructo científico), sino que las personas investigadoras definen qué conocimiento exteriorizado en las interacciones sociales abordarán y cuál no. Así, el discurso es una red de representaciones $y$ asociaciones en torno a un elemento delimitado científicamente.

El discurso es, además, conocimiento considerado como verdadero por un grupo social. Se entiende "considerado como verdadero" a aquel conocimiento que es aceptado por los individuos, es decir, aquellas representaciones $y$ asociaciones que las personas poseen $y$ que guían sus actos $y$, asimismo, son exteriorizadas como propias. Esto conlleva que algunas representaciones $y$ asociaciones no sean aceptadas $y$ se las excluya ${ }^{3}$ (Foucault, 2002), en consecuencia, las excluidas no guían los actos (voluntarios) ni mucho menos son exteriorizadas como propias ¿Qué hace que unas representaciones sean aceptadas y otras, excluidas? Como anteriormente se señaló, las representaciones $y$ asociaciones previas de las personas y su relación con las nuevas construidas en la memoria de trabajo guían el proceso de almacenamiento de nuevas representaciones $y$ asociaciones en la memoria a largo plazo. De esta manera, es el mismo conocimiento de los individuos el encargado de aceptar o excluir nuevas representaciones $y$ asociaciones. Por esto, Jäger (2004) afirma que un conocimiento sea tomado por

2 Un discurso también puede ser delimitado según criterios sociales (de grupos sociales distintos a los científicos) no necesariamente científicos, por ejemplo, el discurso sobre un lenguaje verbal (una lengua) que poseen hablantes particulares (sobre la lengua de inmigrantes, de otras clases sociales o sobre la propia). Sin embargo, al momento de su análisis, se tomarán criterios científicos para utilizar esa delimitación o no.

Excluir no quiere decir únicamente que esas representaciones se eliminen o que no existan, sino que se pueden poseer, pero no son las que guían los actos ni las que se exteriorizan como propias (se podrían exteriorizar como propias de otras personas). verdadero es efecto del discurso mismo (para Jäger el discurso es todo el conocimiento societal acumulado), o sea, el mismo conocimiento de las personas guía qué se acepta o no. Sin embargo, el discurso no solo es considerado como verdadero por una persona, sino, por un grupo social. Cada grupo social delimita qué representaciones $y$ asociaciones son aceptadas. Por esto, podrían existir tantos discursos como grupos sociales.

Un grupo social comparte conocimientos en la medida en que sus miembros llevan a cabo exteriorizaciones (actos físicos o lingüísticos) similares; solo a partir de esto se puede afirmar que un conjunto de representaciones es considerado como verdadero dentro de un grupo social. Así, un discurso se comparte dentro de un grupo social y corresponde a las representaciones $y$ asociaciones manifestadas explícita (perceptibles) e implícitamente (representaciones $y$ asociaciones no perceptibles, pero que se suponen necesarias para realizar los actos) en las exteriorizaciones ${ }^{4}$ de sus miembros.

Dentro de las exteriorizaciones, los actos lingüísticos desempeñan un rol fundamental, ya que, junto con lo visual, constituye el principal instrumento para el pensamiento, incluso, como señala Roth (2003), hay quienes afirman que sin lengua no habría pensamiento (reflexión): cuando se reflexiona sobre el propio conocimiento, se utiliza el lenguaje verbal, incluso, se puede referir mediante este a representaciones mentales no verbales. De esta manera, se hace un paralelo entre las representaciones mentales verbales y los actos lingüísticos, por lo cual cuando se analiza el conocimiento humano o el discurso, se prefiere estudiar exteriorizaciones lingüísticas en lugar de otros tipos de $\operatorname{actos}^{5}$. Lo anterior provoca que en muchas ocasiones se confunda discurso con actos lingüísticos, sin embargo, como se señaló, el discurso es

$4 \quad$ Esto no significa que los miembros de un grupo social realizan siempre las mismas exteriorizaciones ni que no lleven a cabo actos contradictorios.

5 Se estudian otros tipos de actos, sin embargo, se utiliza el lenguaje verbal como instrumento explicativo de esos actos (por ejemplo, se consulta a los actores por qué realizaron un acto particular). 
un conjunto de representaciones $y$ asociaciones $y$, como tales, determinan y se manifiestan en todo tipo de exteriorizaciones y no exclusivamente en las verbales.

Si bien, el científico delimita qué conocimiento exteriorizado $y$, por ende, con qué exteriorizaciones trabajará, difícilmente se encontrará con un discurso puro, es decir, con una red de representaciones que se refieran de forma exclusiva a un algo. Cada vez que se lleva a cabo actos, se requiere de un conjunto de diferentes representaciones sobre diversos estímulos, por lo cual, las exteriorizaciones se encuentran determinadas por una amplia gama de representaciones. Asimismo, las mismas representaciones o nodos pueden determinar $y$ manifestarse en diversos tipos de actos, los que no tienen, necesariamente, una (clara) relación entre sí en la vida social. Por esto, se afirma que un discurso nunca se encuentra aislado, por el contrario, siempre tendrá interrelaciones con otros, lo cual constituye una red discursiva. Se considera que entre estos discursos habría una relación dialéctica, por cuanto se determinarían mutuamente, además, tensiones y afinidades $y$, en consecuencia, unos más influyentes (dominantes) que otros.

Por otro lado, se debe resaltar el carácter histórico y, por lo tanto, dinámico de los discursos. Los grupos sociales con el pasar del tiempo cambian sus actos (por ejemplo, formas de comer, de construir sus hogares, de vestirse, de hablar), lo que quiere decir que ha habido un cambio en el conocimiento (las representaciones mentales determinan los actos). De este modo, se observa el carácter histórico del discurso y, además, muestra su dinamismo, ya que se encuentra en un constante proceso de transformación, al igual que el conocimiento individual. Este dinamismo también se presenta a nivel de la red discursiva, debido a la relación dialéctica y a las tensiones $y$ afinidades entre discursos, que hacen que se modifique su organización. De esta manera, se afirma que el discurso y la red discursiva son tanto una estructura como un proceso. Son una estructura en la medida en que se puede identificar una organización interna de representaciones y asociaciones en un momento histórico particular; y son un proceso, ya que se encuentran en constante construcción (por su dinamismo).

Por último, debido a la interrelación entre grupos sociales (contacto entre distintos discursos), a la posibilidad de que una persona pertenezca a más de un grupo social (poseer diferentes discursos) y al constante proceso de transformación (abierto al cambio), el discurso puede tener representaciones o nodos contradictorios entre sí, lo que se manifiesta en distintos tipos de actos en variados contextos situacionales. Estas contradicciones no constituyen "errores" o "debilidades" del discurso ni tampoco pruebas de que un discurso no se encuentra bien delimitado (labor científica), por el contrario, son evidencias de la riqueza y el dinamismo del conocimiento humano.

\section{RELACIÓN LENGUAJE-DISCURSO}

Se identifican tres tipos de relaciones entre el lenguaje como realización y el discurso como conocimiento compartido tomado como verdadero. En primer lugar, se encuentra el discurso que existe sobre el mismo lenguaje, es decir, todo el conjunto de representaciones y asociaciones sobre el lenguaje (qué es el lenguaje, cómo se realiza, sobre los recursos a disposición, sobre el género, el modelo o el tipo de texto, entre otras) que se manifiesta en actos: el discurso sobre el lenguaje. A partir de esto, se afirma que toda realización de un lenguaje se guía (en parte) por el discurso sobre el lenguaje que tenga la persona. Un ejemplo ${ }^{6}$ de acercamientos al discurso sobre el lenguaje (o parte de este) lo constituyen los trabajos que abordan las valoraciones de hablantes de un lenguaje verbal particular (por ejemplo, sobre una variante particular del español de Costa Rica) sobre las realizaciones propias (el español de Costa Rica) o ajenas (por ejemplo, el español de Nicaragua).

El segundo tipo de relación se da entre realizaciones lingüísticas y otros discursos (distintos al discurso sobre el lenguaje realizado).

$6 \quad$ Esto es solo un ejemplo y no hay que reducir el discurso sobre el lenguaje a estos aspectos, ya que para nosotros, este discurso abarcaría también el conocimiento sobre los actos lingüísticos mismos. 
El lenguaje es un instrumento-medio que permite el diálogo intersubjetivo (la comunicación), ya que a través de sus realizaciones tanto el productor como el perceptor establecen una relación entre materialidad y representaciones $y$ asociaciones, de manera tal que esa materialidad se toma como representante de representaciones $y$ asociaciones. De esta forma, se pueden considerar las realizaciones lingüísticas como las exteriorizaciones (actos) básicas del conocimiento, por cuanto su función es establecer esa relación entre representaciones y asociaciones $y$ materialidad, con el fin de exteriorizar conocimiento. Otros actos de las personas, si bien exteriorizan conocimientos, sus realizaciones tienen otros objetivos. Por esto, el lenguaje constituye el medio por excelencia para la manifestación de los discursos, pero no el único.

La tercera y última relación que se destaca se refiere a la que se da entre discurso sobre el lenguaje $y$ otros discursos. Esta interrelación es parte de esa red discursiva, de la cual participa todo discurso. En esta relación, por ejemplo, el conocimiento sobre qué realización lingüística ${ }^{7}$ se puede llevar a cabo (qué género textual) o qué recursos se utilizarán (qué estilo, palabras, estructuras sintácticas) determinará la manifestación (cómo se exteriorizan) de los otros discursos; y estos últimos, a su vez, por su organización interna (qué representaciones $y$ asociaciones son más importantes) pueden determinar la estructura temática de la realización lingüística o la utilización de diferentes recursos lingüísticos (por ejemplo, para realzar). Así, se observa que, al abordar un discurso determinado a partir de su manifestación lingüística, no se debe dejar de lado la realización lingüística en sí y como esta determina ese discurso en estudio.

$7 \quad$ En coherencia con la postura del investigador, no se afirma que (todas) las personas tengan un conocimiento consciente sobre las realizaciones lingüísticas, sino que deben poseer representaciones y asociaciones mínimas que les permitan llevar a cabo esos actos.

\section{DISCAPACIDAD}

A través de la historia han (co)existido diferentes formas de entender la discapacidad (Palacios, 2008; Egea y Sarabia, 2004; Pantano, 2009; López, 2006; Dannenbeck, 2007; Dederich, 2007; etc): desde posturas religiosas hasta sociales, pasando, por supuesto, por biológicas. Si bien algunas conceptualizaciones no han llegado (claramente) hasta estos días, muchas de ellas conviven en nuestras sociedades (Gray, 2009). En el marco de este trabajo, se destaca la perspectiva conocida como modelo social de la discapacidad (Palacios, 2008). Esta postura entiende la discapacidad como:

...la desventaja o restricción de actividad, causada por la organización social contemporánea que no considera, o considera en forma insuficiente, a las personas que tienen diversidades funcionales, y por ello las excluye de la participación en las actividades corrientes de la sociedad (Palacios, 2008, p.123).

De esta manera, se observa que la discapacidad no es una "deficiencia" ${ }^{8}$ física, mental, intelectual o sensorial, sino una construcción social que genera que algunas personas se encuentren en desventaja en comparación con otras para llevar a cabo su vida en sociedad. A pesar de que este modelo ha recibido varias críticas (Gray, 2009), el estudio se apoya en este, ya que sirvió de base para la redacción de la Convención Internacional sobre los Derechos de las Personas con Discapacidad.

A partir de lo expuesto hasta el momento, se afirma que el análisis (crítico) del discurso sobre la discapacidad (y la red discursiva en la que se manifiesta) constituye una tarea fundamental para comprender la discapacidad. La discapacidad se entiende como una situación de desventaja producida por los actos y productos sociales basados en el conocimiento de un grupo social (mayoritario o minoritario) sobre

8 En español, el término "deficiencia" puede tener una connotación negativa, sin embargo, se utiliza porque los teóricos sobre la discapacidad recurren a este y por razones de estilo. También se usará "diversidad funcional" con la misma acepción. 
las personas con alguna "deficiencia" física, mental, intelectual o sensorial. En otras palabras, el discurso sobre la discapacidad (junto a otros discursos de una red discursiva manifestada en una situación particular) guía la actuación de las personas hacia quienes tienen discapacidad. De esta manera, la descripción y explicación de este discurso aportaría elementos relevantes para identificar los componentes (representaciones $y$ asociaciones) que poseen los individuos de una sociedad que originan actos y prácticas sociales discriminatorias y excluyentes hacia las personas con discapacidad.

En cuanto a la relación del discurso sobre la discapacidad manifestado a través de actos lingüísticos que no sean respuestas a encuestas, se carece de estudios académicos en Costa Rica $y$, al parecer, en gran parte de los países hispanohablantes ${ }^{9}$. Por esto, se hace referencia al trabajo de Gray (2009), en el cual, se identifican tres "narrativas ${ }^{10}$ " frecuentes e interrelacionadas: de asimilación, con la que se intenta borrar la deficiencia física y se la presenta como una tragedia, la cual se debe curar; de hipenación (hyphenation), se refiere a la construcción en la que se incorpora a las personas con discapacidad dentro de la sociedad, sin embargo, estas cargan todavía con el estigma, de manera que se las ve como protagonistas de una historia de superación; multicultural, esta narrativa considera las deficiencias como parte de una sociedad pluricultural como una diferencia, así no conlleva las valoraciones afectivas de tragedia o "triunfo" de las otras narrativas, no obstante, mantiene una jerarquía entre las habilidades de los individuos, de manera que se categorizan a las personas según sus habilidades ${ }^{11}$. Los planteamientos de esta autora

$9 \quad$ No se ha encontrado bibliografía sobre este objeto de estudio.

10 La autora utiliza el término "narrativas" (narratives), sin embargo, se lo podría equiparar al concepto de discurso que se utiliza en este artículo, en cuanto son construcciones simbólicas (conocimiento) materializadas.

11 Como bien afirma Gray (2009), podría suceder que para un grupo de personas una deficiencia física con sus correspondientes habilidades se ubique en un lugar superior de la estructura jerárquica. servirán de apoyo argumentativo para la explicación y crítica de los resultados obtenidos en este análisis.

\section{CARACTERÍSTICAS DEL CORPUS}

En este trabajo, se analizan fragmentos ${ }^{12}$ de dos actas de sesiones plenarias de la Asamblea Legislativa de Costa Rica, en los cuales se discute la aprobación de la Convención Internacional sobre los Derechos de las Personas con Discapacidad: Acta de la sesión plenaria nro. 027 (páginas 33-62), del martes 27 junio de 2008, en la que se dio el primer debate sobre la Convención, y Acta de la sesión plenaria nro. 055 (páginas 21-52), del 7 de agosto de 2008, donde se llevó a cabo el segundo debate y su aprobación.

Las actas de las sesiones plenarias son el registro escrito de las discusiones e intervenciones que se dan en las asambleas plenarias del Congreso de Costa Rica. Si bien, en estas sesiones se deberían dar las discusiones y negociaciones entre los diputados y las diputadas, la realidad es diferente, ya que los intercambios argumentativos entre este grupo o las personas asesoras se realizan en las comisiones y en los pasillos y oficinas de la Asamblea Legislativa. Por este motivo, se considera que el Plenario debe ser abordado como el lugar en que los diputados $y$ diputadas y los partidos (cuando hablan en nombre de un partido) manifiestan sus posiciones públicamente. Es decir, las intervenciones, en general, están dirigidas a la ciudadanía y a la historia.

Tanto la publicidad como el registro histórico tienen repercusiones en las realizaciones lingüísticas. Es así como, por ejemplo, la mayoría de las intervenciones han sido preparadas ya sea en forma de un texto o de un listado de ideas a desarrollar y son escasas las intervenciones espontáneas. Además, estas realizaciones lingüísticas están en la mira de la evaluación pública, por lo cual se evitan expresiones (por ejemplo, vulgares o populares), temas $\mathrm{u}$ opiniones que puedan ser sancionadas

12 No se tomó en cuenta las discusiones sobre otros temas. 
socialmente, de ahí que prime lo "políticamente correcto13" (por supuesto, dentro de cada perspectiva política). Asimismo, como lingüistas no se puede dejar pasar por alto el hecho de que las actas corresponden a un registro escrito de un texto originario oral, o sea, es una transcripción que utiliza los signos propios de la escritura y no un sistema de transcripción más preciso, por lo cual, se pierden elementos fundamentales para reconstruir las intervenciones originales ${ }^{14}$.

Los aspectos anteriores provocan que las actas como género textual sean de un interés particular, por cuanto deberían escasear o ser muy sutiles todas las construcciones excluyentes o discriminatorias contra cualquier grupo social, pero, en especial, en contra de las personas con discapacidad, ya que se lo considera un grupo desprotegido social y jurídicamente. De esta manera, se requiere de un análisis fino (ver Vergara s.f.a y s.f.b) para evidenciar cualquier referencia que "atente" contra las personas con discapacidad.

\section{LA DISCAPACIDAD EN LAS ACTAS DE LA ASAMBLEA LEGISLATIVA DE COSTA RICA}

En esta sección se presentan, explican y critican algunos de los componentes del discurso sobre la discapacidad excluyente $y$ discriminatorio manifestado en las actas analizadas. No se pretende exponer todos los resultados, sino (de)mostrar que existe este discurso, incluso, en géneros textuales tan normados como estas actas. Cabe señalar que no se relacionará a los emisores con los enunciados y, por ende, tampoco se distinguirán posturas políticas (o ideológicas), debido a motivos de espacio, pero estos aspectos se abordarán en otros textos académicos.

13 Por "políticamente correcto", se entienden aquellos actos (en especial, lingüísticos) que se realizan para no recibir sanciones sociales tácitas según unos discursos dominantes en contra de diversas formas de discriminación y exclusión.

14 A esto cabe sumar que las actas deben ser aprobadas por el Plenario, por lo cual podrían ser corregidas (aunque no deberían eliminarse muchos elementos).

\section{DISCAPACIDAD COMO CARACTERÍSTICA DE LAS} PERSONAS

Según el modelo social de la discapacidad, el cual fundamenta la Convención Internacional sobre los Derechos de las Personas con Discapacidad, la discapacidad se origina de la interrelación entre una deficiencia (o diversidad funcional) $y$ el entorno situacional $y$ social en que se desenvuelve una persona. En otras palabras, la discapacidad no es la deficiencia física, sensorial, intelectual o mental de las personas, sino esa construcción a partir de una deficiencia y el entorno. Esto tiene suma relevancia, por cuanto lo social (el entorno) es parte de la discapacidad $y$, por ende, la discapacidad no corresponde a una característica individual. A pesar de esto y de que las intervenciones en el Plenario, en su mayoría, son planificadas, se observa que diputados y diputadas entienden la discapacidad como propiedad de las personas y no como esa interrelación entre entorno $y$ deficiencia.

(1) ...discapacidad es un problema que la misma sociedad agrava poniendo barreras legales y sociales... (acta nro. 027, p. 35).

(2) Las discapacidades son múltiples. Algunas personas tienen una discapacidad física, producto de un accidente de tránsito, otras personas nacen con una discapacidad mental... (acta nro. 055, p. 45).

En el ejemplo 1, se observa una distinción entre discapacidad (como problema) y las barreras del entorno, por lo cual para este diputado en el concepto de discapacidad no entra el entorno social, sino que corresponde a una propiedad de las personas (con deficiencias). Lo anterior se hace más evidente en el fragmento 2 , ya que el hablante se refiere a múltiples discapacidades $y$, luego, ejemplifica con una física $y$ otra mental sin mencionar el entorno, con lo que evidencia que equipara "discapacidad" con "deficiencia".

La equiparación entre discapacidad y deficiencia (o diversidad funcional) tiene como consecuencia que se considera la discapacidad 
como una característica de unas personas y no como una interrelación con el entorno. De esta manera, se muestra que, a pesar de ser textos planificados $y$ de discutirse sobre la Convención $^{15}$, el modelo social de la discapacidad no se encuentra completamente insertado en el discurso.

\section{DISCAPACIDAD COMO PROBLEMA}

En el punto anterior, se mostró como se considera que la discapacidad es una característica de las personas y no una construcción. A continuación, se muestra cómo se valora esa propiedad dentro del discurso sobre la discapacidad.

(1) ...discapacidad es un problema que la misma sociedad agrava poniendo barreras legales y sociales... (acta nro, 027, p. 35).

(3) ...sobre un problema ${ }^{16}$ tan importante para más de seiscientas mil personas ${ }^{17}$ en nuestra patria... (acta nro. 027, p. 43).

En estos ejemplos se observa, además que la discapacidad es una propiedad de unas personas determinadas, que se la valora como un problema, es decir, negativamente. De esta manera, se asemeja a lo que caracteriza a la "narrativa de la asimilación", en la que la deficiencia de las personas se ve

15 Se esperaría que si se discute en la Asamblea Legislativa sobre la Convención Internacional sobre los Derechos de las Personas con Discapacidad, se maneje la terminología que da fundamento a ese texto.

16 Si bien, en el fragmento citado no es clara la referencia a la discapacidad como propiedad de las personas, esto se evidencia en el siguiente enunciado del mismo diputado: "De todos es conocido que las personas con discapacidad sufren una serie de obstáculos, (sic) físicos y culturales excluyentes..." (acta nro. 027, p. 45).

17 Se estima que en Costa Rica hay 60000 personas con discapacidad. Se cree que el diputado se equivocó, debido a que mezcló el número de personas en el ámbito mundial y nacional, ya que la OMS calcula que hay 600 millones de personas con discapacidad en el mundo. como una tragedia o un elemento negativo, el cual - dentro de lo posible - se debería curar o evitar (por ejemplo, campañas para evitar la conducción de vehículos motorizados bajo la influencia del alcohol mediante la ostentación de personas en sillas de ruedas debido a accidentes de tránsito).

Conceptualizar la discapacidad o, mejor dicho, las diversidades funcionales (deficiencias físicas, mentales, intelectuales o sensoriales) como algo negativo (tragedia) conlleva una estigmatización de un grupo social, el cual se representa como "víctima". Esta estigmatización provoca que estas personas se encuentren siempre en un lugar inferior dentro de la estructura jerárquica social, por cuanto "lamentablemente" no tendrían las mismas capacidades (habilidades) que las personas "normales". En otras palabras, el hecho de no poseer las habilidades de las personas "normales" se valora negativamente. Con este discurso, se hace difícil que estas personas puedan incluirse en igualdad de condiciones respecto a las "normales" en la sociedad, porque cargarán siempre con el estigma de poseer algún tipo de deficiencia, la cual es valorada como problema por otros grupos sociales $y$-posiblemente_- por las mismas personas con diversidad funcional. Esta construcción se manifiesta también en el uso de diminutivos:

(4) ...Yo creo que esta personitas [adultos con discapacidad] tienen derecho a ser, no solamente independientes, sino autosuficientes... (acta nro. 055, p. 31).

Se ve como este diputado utiliza un sustantivo en diminutivo para referirse a adultos. Con esto, se evidencia una infravaloración de estos individuos, por cuanto no se los denomina "personas", sino se apela al diminutivo (gradación apreciativa), lo cual reflejaría que su situación (poseer algún tipo de deficiencia) es considerada como un problema o tragedia. Se puede afirmar que se apela, de alguna forma, a la lástima.

\section{HISTORIAS DE SUPERACIÓN: SOBREVALORACIÓN} DE LAS PERSONAS CON DISCAPACIDAD

Directamente relacionado con la construcción anterior, se encuentra la sobrevaloración 
de las personas con diversidades funcionales que llegan a ocupar un lugar destacado en la estructura social. Se hace referencia a aquellas "historias de superación", en las cuales, se resalta como una persona con una deficiencia logra alcanzar determinados objetivos sociales (puestos públicos, reconocimiento social) a pesar de los obstáculos del entorno.

(5) ...que él [diputado Óscar López] que ha tenido que experimentar ese esfuerzo personal, esa superación, [...] me impresionó como esa experiencia personal del diputado Óscar López y esa capacidad, esa tenacidad de superación... (acta nro. 027, p. 44).

(6) ....y aquí en el Plenario legislativo lo vive todos los días un compañero nuestro, el compañero Óscar López, cuya superación es un ejemplo... (acta nro. 027, p. 48).

En estos fragmentos, se ve como dos diputados destacan el logro de Óscar López ${ }^{18}$, su historia de vida, y la "superación" de las barreras del entorno hasta llegar a ser diputado de Costa Rica. Como se afirmó, esta construcción se relaciona directamente con la infravaloración de las diversidades funcionales, por cuanto solo se puede resaltar la historia de Óscar López, si se comparte que esa deficiencia física es un problema para desenvolverse socialmente o para llegar donde llegó esta persona (Gray, 2009). En resumen, toda sobrevaloración de este tipo de historias implica una infravaloración de las personas con diversidad funcional ${ }^{19}$.

18 El diputado Óscar López es una persona ciega o con visión reducida.

19 Un ejemplo de estas construcciones son las historias que se presentan en distintos lugares del mundo con motivo de una Teletón (por ejemplo, en Chile y EE.uU.).

\section{PERSONAS CON DISCAPACIDAD: GRUPO EXCLUIDO DE LA SOCIEDAD}

Otro aspecto interesante de este discurso es que se considera a las personas con discapacidad como un grupo social externo a la sociedad, por un lado, se encuentra la sociedad $y$, por otro lado, esta población. Esto no llamaría la atención, ya que esta idea ha sido un componente del discurso sobre la discapacidad dentro de los mismos sectores que defienden los intereses de las personas con discapacidad. No obstante, una cosa es pensar que en la actualidad este grupo está, en su mayoría, excluido, y otra muy distinta es reafirmar esto presentando a la sociedad como un actor agente, asignándole responsabilidades, mientras que las personas con discapacidad aparecen como excluidos y como actores pasivos, beneficiarios. Esto último evidencia que para ese discurso las personas con discapacidad están y seguirán estando excluidas de la sociedad.

(7) ..este grupo de individuos [personas con discapacidad] que requieren [...] la protección y el respeto de la sociedad (acta nro. 027, p. 34).

(8) ..personas [personas con discapacidad] que tienen los mismos derechos que tenemos los ciudadanos de este país... (acta nro. 027, p. 35).

(9) ...hago un llamado respetuosos (sic) a los señores diputados $y$ diputadas, al sector público y al sector privado, $y$ a la sociedad en su conjunto, en el sentido de que debemos trabajar para una eficaz $y$ eficiente inclusión e inserción de las personas con discapacidad... (acta nro. 27 , p. 56).

En el fragmento 7, se observa la clara separación entre las personas con discapacidad $y$ la sociedad, donde esta última es la encargada de actuar en beneficio de ese grupo excluido. Además, esta exclusión se hace más evidente cuando la misma diputada (8) hace la distinción 
entre ciudadanos y personas con discapacidad. Si bien es cierto que afirma que ambos grupos tienen los mismos derechos, no considera a las personas con discapacidad como parte de la ciudadanía de este país. En el ejemplo 9, otra diputada también enuncia esta separación entre sociedad $y$ personas con discapacidad, sin embargo, se diferencia en que hace un llamado de atención sobre la situación actual, en la que las personas con discapacidad se encuentran excluidas. De esta manera, se afirma que, por un lado, en los dos primeros casos (7 y 8), la exclusión social de las personas con discapacidad pareciera una condición de ese grupo, es decir, no una situación temporal y modificable, sino como algo estable y perdurable. Por otro lado, en el ejemplo 9 se hace referencia a una situación actual, la cual podría modificarse si se logra "una eficaz y eficiente inclusión e inserción de las personas con discapacidad" (acta nro. 027, p. 56); sin embargo, no son estas las personas llamadas a actuar, sino la sociedad (en la que no están incluidas las personas con discapacidad), por lo que se representa a las personas con discapacidad como no activas $y$ dependientes, aspectos que se desarrollan en el siguiente apartado.

\section{PERSONAS CON DISCAPACIDAD: PERSONAS NO ACTIVAS Y DEPENDIENTES}

En estrecha relación con el apartado anterior, se ha encontrado un componente del discurso sobre la discapacidad que - quizáscorresponda al principal aspecto para lograr una "real" inclusión de las personas con diversidad funcional: la "inmovilidad" y la dependencia de las personas con discapacidad. En distintos pasajes de las actas se encuentran referencias o llamados a autoridades y personas sin discapacidad a actuar para lograr la inclusión de este grupo social. Esta postura implica que las personas con discapacidad no pueden hacer nada (actuar) respecto a su propia vida $y$ que dependen de los actos de otros individuos para su desarrollo personal.

Si bien es cierto que, por un lado, hay personas con diversidad funcional que requieren de la colaboración de alguna persona para realizar algunas actividades y que, por otro lado, es necesario que los otros miembros de una sociedad actúen para lograr la inclusión de las personas con discapacidad, no se le debe asignar toda la responsabilidad a las personas sin discapacidad, ya que esto es contrario a la autonomía e independencia que debe haber para una "verdadera" inclusión. Si los individuos son dependientes, entonces se encuentran en una posición jerárquica inferior respecto de quienes dependen; de esta manera, a priori, la igualdad entre los miembros de una sociedad (si es que se logra cierto grado de inclusión) no puede existir.

(9) ...hago un llamado respetuoso a los señores diputados $y$ diputadas, al sector público $y$ al sector privado, $y$ a la sociedad en su conjunto, en el sentido de que debemos trabajar para una eficaz y eficiente inclusión e inserción de las personas con discapacidad... (acta nro. 027, p. 56).

(10) ...creo fundamental que recapacitemos y logremos la integración [...] de las personas con discapacidad... (acta nro. 027, p. 58).

Tanto en el ejemplo 9 como en el 10, se hace un llamado a la población sin discapacidad para que actúe y logre la inclusión de las personas con discapacidad. Es ese primer grupo el actor y responsable de la inclusión. De esta manera, las personas con discapacidad no son partícipes de ese proceso $y$ dependen del actuar de otros. Estos ejemplos ponen de manifiesto esa representación de inactividad y dependencia propias de las personas con discapacidad. Esto refuerza la "imagen" de víctima que se construye a partir de la consideración de las deficiencias físicas, mentales, sensoriales e intelectuales como una tragedia. Con estos componentes del discurso sobre la discapacidad, nuevamente, se infravalora a las personas con discapacidad, por cuanto necesitan de otras para vivir y se imposibilita su autonomía e independencia.

A pesar de que los textos analizados pertenecen, en general, a un género textual planificado y normado por lo "políticamente 
correcto", se encontró un discurso sobre la discapacidad que, en primer lugar, considera la discapacidad como una característica física, mental, intelectual o sensorial de un grupo de personas, lo cual se aleja del modelo social de la discapacidad, base de la Convención. En segundo lugar, esa característica se valora como una tragedia, como algo negativo que le sucede a ese grupo de personas. Esto posiciona a las personas con discapacidad como víctimas y las estigmatiza. Tercero, una consecuencia de la representación como víctimas es sobrevalorar a las personas con discapacidad que ocupan un lugar destacado socialmente. Esta sobrevaloración evidencia una infravaloración de las personas con discapacidad, ya que solo así se entiende que el hecho de que una de estas personas alcance una posición pública relevante tenga que considerarse como una historia de superación, de triunfo sobre la tragedia. Si las personas con diversidad funcional fueran consideradas en el mismo nivel que una persona "normal", entonces, esas historias no serían destacables. En cuarto lugar, se considera a las personas con discapacidad como un grupo separado de la sociedad, el cual es, por último, inactivo y dependiente. Así, las personas con discapacidad son presentadas como un grupo que siempre necesitará de las otras personas para desarrollarse, por ende, que carece de autonomía e independencia, y que no desempeña ningún rol importante en su proceso de inclusión social.

Todos estos componentes del discurso sobre la discapacidad muestran que existe un discurso excluyente $y$ discriminatorio hacia las personas con diversidad funcional en la sociedad costarricense. Tomando en cuenta que los discursos guían los actos, entonces, se puede afirmar que difícilmente este grupo podrá lograr su inclusión social, ya que se lo infravalora, estigmatiza y no se lo considera como individuos autónomos e independientes, por lo que la no actuación del resto de la ciudadanía sería acorde a estas valoraciones Asimismo, este discurso conlleva la perduración de la discapacidad (según el modelo social de la discapacidad), por cuanto generará barreras en el entorno social (por ejemplo, ¿cómo podría una persona con deficiencia mental o intelectual vivir sola o tomar decisiones económicas?) que impedirá su participación plena en la sociedad.

Cabe señalar que del análisis anterior no se debe interpretar que los diputados y las diputadas de la Asamblea Legislativa hayan manifestado exclusivamente un discurso excluyente $y$ discriminatorio sobre la discapacidad. De hecho, se nota la planificación de las intervenciones, ya que la mayoría de los enunciados corresponden a lo "políticamente correcto" y se acercan al modelo social de la discapacidad (y a los planteamientos de la Convención). Así, se observa, por ejemplo:

(11) ...al reconocer que las personas con discapacidad tienen el derecho de tomar sus propias decisiones, queda claro que son libres. Realmente siempre han sido libres, lo que ha ocurrido es que con las prácticas asistencialistas se les ha obstaculizado el ejercicio de su libertad (acta nro. 027, p.54).

(12) Y decía yo, que toda la sociedad [incluyendo a las personas con discapacidad], en su conjunto, es la que se tiene que educar... (acta nro. 027, p.49).

En el ejemplo 11, un diputado afirma que las personas con discapacidad son libres, postura que es contraria a aquella que plantea que son inactivos y dependientes, ya que esa libertad es la que les permite tomar las propias decisiones, es decir, ser autónomos e independientes. Por su parte, en el fragmento 12 , se incluye a las personas con discapacidad dentro de la sociedad, no son un grupo separado, sino que se lo considera como miembro. Esto muestra la tensión entre diferentes discursos sobre la discapacidad presentes en estos textos $y$ demuestra que los discursos se insertan dentro de una red discursiva (discurso sobre el lenguaje, discurso sobre lo políticamente correcto, discurso sobre la discapacidad según el modelo social, entre otros) que determina su manifestación textual. Por esta razón, se recuerda que el objetivo de este análisis no es mostrar EL discurso sobre la discapacidad que se manifiesta en los textos 
analizados, sino los componentes de un discurso sobre la discapacidad que es excluyente $y$ discriminatorio $y$ que si se presenta en un texto tan planificado y normado socialmente (de acuerdo a lo "políticamente correcto"), significa que existe en la sociedad costarricense $y$, por lo tanto, es compartido por muchos sectores sociales (incluyendo a muchas personas con discapacidad).

\section{A MODO DE CIERRE}

Se considera que el análisis del discurso $y$, en particular, el análisis crítico del discurso, son herramientas importantes para la transformación social, debido a que si se conocen los discursos que guían esos actos excluyentes y discriminatorios, se puede luchar contra ellos. Lo anterior se evidencia del análisis presentado en estas páginas. Se mostró que en Costa Rica existe un discurso sobre la discapacidad $y$, específicamente, sobre las personas con discapacidad excluyente $y$ discriminatorio, el cual ha producido un entorno social que no permite (ni garantiza) la participación de estas personas en la sociedad costarricense.

Asimismo, se toma en cuenta que si se quiere alcanzar una "real" inclusión, se debe transformar ese discurso sobre la discapacidad que comparten varios sectores sociales tanto en Costa Rica como en el mundo, debido a que ese discurso genera todos esos actos y productos sociales que discriminan a las personas con discapacidad. No basta con volver accesible la infraestructura, transporte o la educación, sino llevar a cabo políticas dirigidas en contra de esos componentes. Si se entiende la discapacidad como una construcción a partir de la interrelación entre entorno social y deficiencia física, mental, intelectual o sensorial, entonces, mientras exista este tipo de discurso sobre la discapacidad, siempre habrá discapacidad, por cuanto permanecerán las barreras sociales y actitudinales.

\section{REFERENCIAS}

Asamblea Legislativa de la República de Costa Rica (17/06/2008). Acta de la Sesión Plenaria nro. 027, 33-62.
Asamblea Legislativa de la República de Costa Rica (07/08/2008). Acta de la Sesión Plenaria nro. 055, 21-52.

Busse, D y Teubert, W. (1994). Ist Diskurs ein sprachwissenschaftliches Objekt? Zur Methodenfrage der historischen Semantik. Begriffsgeschichte und Diskursgeschichte: Methodenfragen und Forschungsergebnisse der historischen Semantik, 10-28. Opladen: Westdeutscher.

Convención Internacional sobre los Derechos de las Personas con Discapacidad. ONU. Recuperado de http://www.un.org/esa/ socdev/enable/documents/tccconvs.pdf

Dannenbeck, C. (2007). Paradigmenwechsel Disability Studies? Für eine kulturwissenschaftliche Wende im Blick auf die Soziale Arbeit mit Menschen mit besonderen Bedürfnissen. Disability Studies, Kultursoziologie und Soziologie der Behinderung. Erkundungen in einem neuen Forschungsfeld. Bielefeld: Transcript, 103-125.

Dederich, M. (2007) Körper, Kultur und Behinderung. Eine Einführung in die Disability Studies. Bielefeld: Transcript.

Egea García, C. y A. Sarabia Sánchez. (2004). Visión y modelos conceptuales de la discapacidad. Revista Polibea,(73), 29-42.

Foucault, M. (2002). El orden del discurso. Barcelona: Tusquets.

Gray, C. (2009). Narratives of Disability and the Movement from Deficiency to Difference. Cultural Sociology 3(2),317-332.

Jäger, S. (2004). Kritische Diskusanalyse. Eine Einführung. Münster: Unrast.

Jäger, S. (2003). "Discurso y conocimiento: aspectos teóricos y metodológicos de la crítica del discurso y del análisis de dispositivos". En R. Wodak \& M.Meyer (Eds.). Métodos de análisis crítico del discurso, 61-100. Barcelona: Gedisa.

López, M. (2006). Modelos teóricos e investigación en el ámbito de la discapacidad. Hacia la incorporación de la experiencia personal. Revista Docencia e Investigación. Escuela Universitaria de Magisterio de Toledo, XXXI (16), 215-240. 
Palacios, A. (2008). El modelo social de discapacidad: orígenes, caracterización y plasmación en la Convención Internacional sobre los Derechos de las Personas con discapacidad. Madrid: Cinca.

Pantano, L. (2009). Nuevas miradas en relación con la conceptualización de la discapacidad. Condición y situación de discapacidad. En P. Brogna (Comp.) Visiones y revisiones de la discapacidad (pp.73-97). México: Fondo de Cultura Económica.

Roth, G. (2003). Fühlen, Denken, Handeln. Wie das Gehirn unser Verhalten steuert. Frankfurt am Main: Suhrkamp.

Vergara, A. (2016). El discurso sobre la criminalidad en las noticias televisivas en Costa
Rica. Logos: Revista de Lingüística, Filosofía y Literatura 26 (1), 241-259.

Vergara, A. (s.f.a). El análisis del contenido textual: una propuesta metodológica para el trabajo con historias de vida y grupos de discusión (sin publicar).

Vergara, A. (s.f.b.). El uso de categorías de análisis en los estudios del discurso: una propuesta metodológica para el análisis del discurso de la discapacidad (sin publicar).

Fecha de ingreso: 14/11/2017

Fecha de aprobación: 10/10/2018 
